\title{
COMMUNICATIONS
}

\section{Combination of HP and LP filters for narrow band antialiasing filter}

\author{
Bohumil Brtník ${ }^{1}$
}

\begin{abstract}
The discrete time signal processing requires an anti-aliasing filter at the input and a reconstruction filter at output. Some filters of biquads structure are characterized by a decreasing of the attenuation at high frequencies, caused by the final value of the output resistance of the operational amplifier. In this paper we discuss a design of combined BP filter without mentioned decrease. The proposed filter structure was verified by SPICE simulation.

Keywords: low-pass, band-pass filters structure, real operational amplifier; frequency response, decrease of the attenuation at high frequencies
\end{abstract}

\section{Introduction}

The circuits that process the signal at the discrete time ie switched capacitor circuits, switched currents and digital signal processing circuits, require for their operation an ANTI(?)aliasing filter at the input and a reconstruction filter at its output. If the narrow frequency band is processed, it is possible to decrease thesample frequency. These anti-aliasing filters should have the band pass properties. However, the real properties of operational amplifiers used in active RC (ARC) filters will modify the monotone-decreasing frequency response.

Some biquad filters structures are characterized by a decrease of attenuation at higher frequencies [1], [2]. This occurs only with filters of even orders, $i e$ for biquads, too.

The cause of these attenuation losses can be explained, see Fig. 2, in a case of a second order low-pass filter. The ideal frequency response of a low-pass filter would be continuous decreasing with $-40 \mathrm{~dB} / \mathrm{dec}$ slope. The finite value of the transient frequency $\omega_{\mathrm{T}}$ of the used operational amplifier leads to the break of this (ideal) slope. The result is a less attenuation in the stopband for around the transient frequency $\omega_{\mathrm{T}}$.

The sample frequency $f_{S}$ is determined by Shannon theorem

$$
f_{\mathrm{s}}=2 f_{\max }
$$

where, $f_{\max }$ is maximum frequency component of the input signal. If the signal spectra does not contain very low frequencies, but starts at $f_{\min } i e$ for this narrow-band signal the sampling frequency can be decreased

$$
f_{\mathrm{s}}>\frac{2 f_{\max }}{\operatorname{int}\left(\frac{f_{\max }}{f_{\max }-f_{\min }}\right)} .
$$

The filtering can be done using only $\mathrm{BP}$ or HP and LP filter, the useful criterium is $[3,4]$

$$
\frac{f_{\max }}{f_{\min }}\left\{\begin{array}{ll}
<2 & \mathrm{BP} \\
\geq 2 & \mathrm{HP}+\mathrm{LP}
\end{array} .\right.
$$

Consider signal with $f_{\min }=5 \mathrm{kHz}$ and $f_{\max }=10 \mathrm{kHz}$, in this case $f_{s}=10 \mathrm{kHz}$ according to (2), while by (1) we get $f_{s}$ as much as twice higher. The BP filter can be realised as combination an HP + LP filter. Note that, (3) gives $f_{\max } / f_{\min }=2$

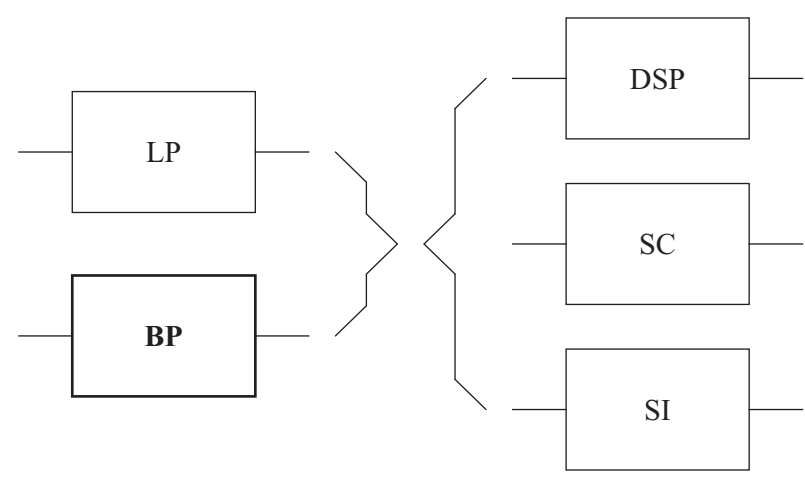

Fig. 1. The antialiasing filter in circuit

One of favorite structures is the Sallen-Key filter (SK). Its disadvantage is decrease of attenuation over transient frequency of the operational amplifier, as mentioned above, [5]. Let us consider a 4 th order filter. Because $f_{\mathrm{c} 2} / f_{\mathrm{c} 1}=2$, the 4 -th both used filters (HP and LP) must be of 4 -th order. Each of them consists of two biquads (2-nd order), as depicted in Fig. 3.

Finite transient frequency of operational amplifiers is the reason of a decrease at the higher frequencies, $i e$ over $f_{\mathrm{T}}$, as shown in Fig. 4, where the capacitors are replaced

${ }^{1}$ University of Pardubice, Faculty of the Electronics and Informatics Czech Republic, bohumil.brtnik@upce.cz 


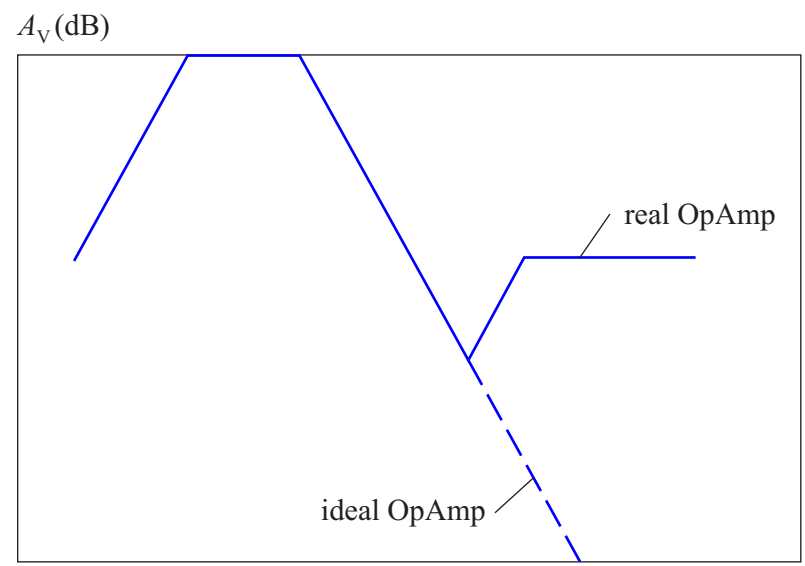

$f(\mathrm{~Hz})$

Fig. 2. The Bode plot of SK-SK BP filte

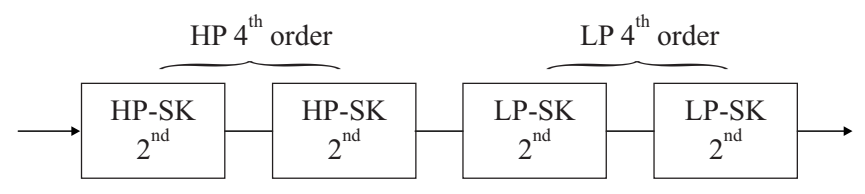

Fig. 3. The filter structure

by shorts [6-8]. In this condition the circuit is a frequency independent voltage divider, unfortunately $V_{\text {out }}>0$

To suppress unwanted output, it is sufficient to replace one of LP-SK biquads by the Huelsmann biquad (LP-H). The reason is the shorting effect by the input capacitor in Huelsmann structure. In this case, the last circuit in cascade is "excited" by a zero voltage, as depicted in Fig. 5. Notice that if the first LP-SK biquad was be replaced by LP-H structure (the second one remaining LP-SK) there would be noise from the H-LP and SK-LP structure at the circuit output. But, if the second one LPSK is replaced by H-LP structure, then the output noise is due to H-LP structure only.

\section{Design of the described combination filter}

Let the Butterworth filter be choosen for example. Then the design steps are as follows:

- The capacitances in HP-SK $2^{\text {nd }}$ order filters $\left(C_{1}=\right.$ $C_{2}=C$ in Fig. 4-5) were chosen to be $100 \mathrm{nF}$.

- The Butterworths approximation coefficients as well as the resistors are calculated from coefficients: $F_{0}, Q$ (see Tab.1) as: $b=1 / F_{0}^{2}=1$ and $a=\sqrt{b} / Q=$ $1.845 ; 0.765$ and $R_{1}=1 /(2 \omega b C)=159 \Omega, R_{2}=$ $1 /(\omega a C)=344 \Omega ; 831 \Omega$ for Section 1 and Section 2, respectively. See Fig. 6 the upper part.

- The capacitances in LP-SK $2^{\text {nd }}$ order filters $\left(C_{4}\right.$ in Fig. 4-5) were chosen to be $1 \mathrm{nF}$.

- For biquads (in Fig. 4 - Sections 3,4; or in Fig. 5 - Section 3 ) there are coefficients $m=1 / Q^{2}=3.41 ; 0.585$ leading to capacitances

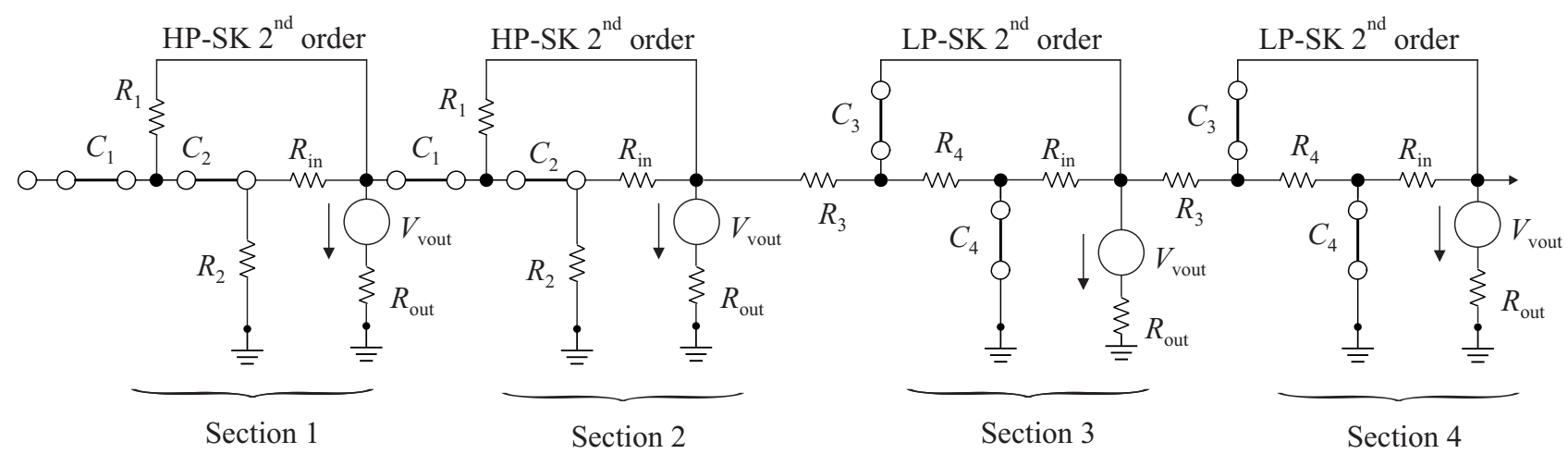

Fig. 4. BP filter HP-SK + LP-SK over operational amplifier transient frequency $f_{\mathrm{T}}$

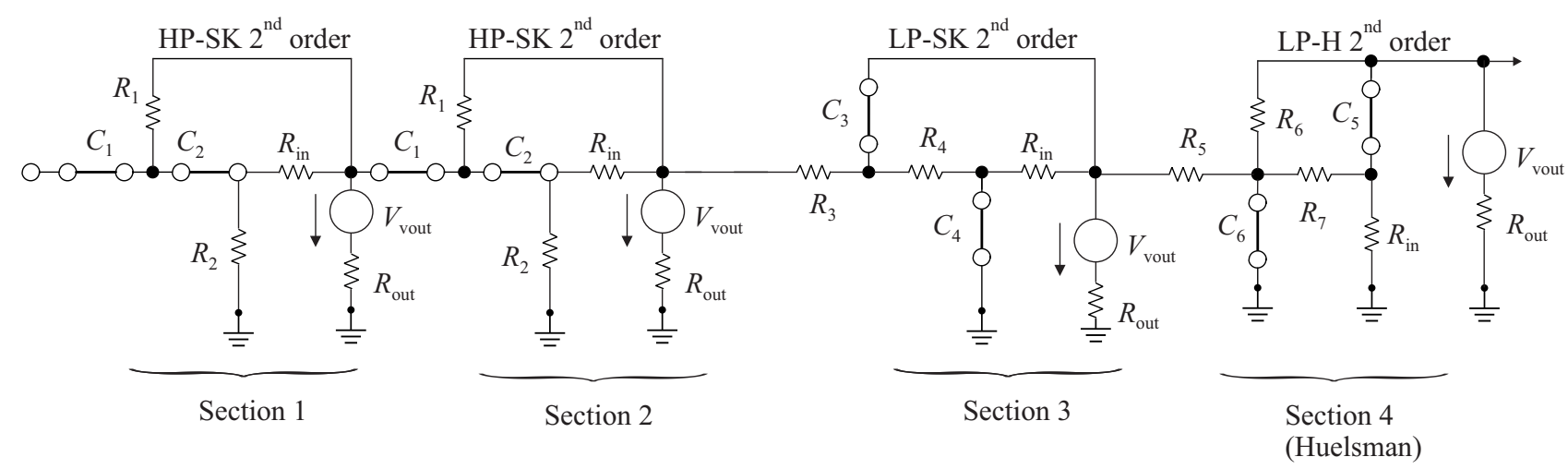

Fig. 5. BP combination filter HP-SK-SK + LP-SK-H over operational amplifier transient frequency $f_{\mathrm{T}}$ 

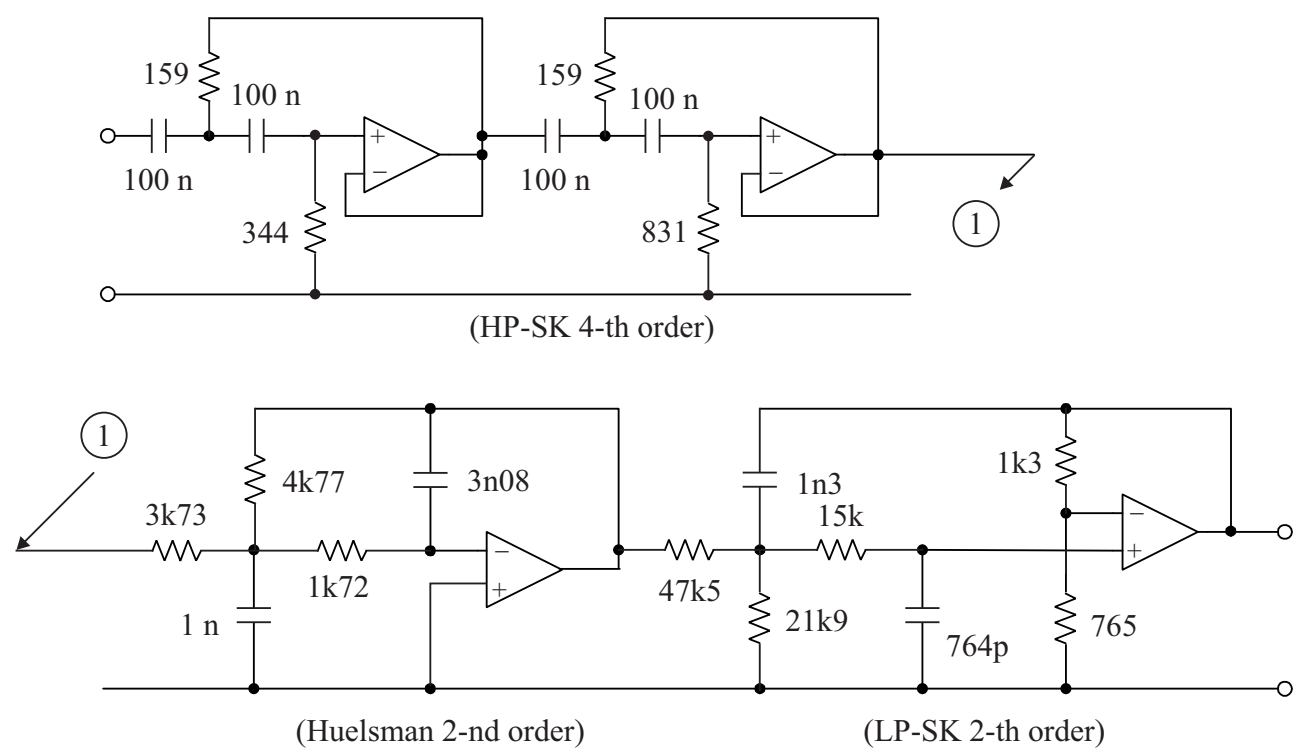

Fig. 6. The designed circuit

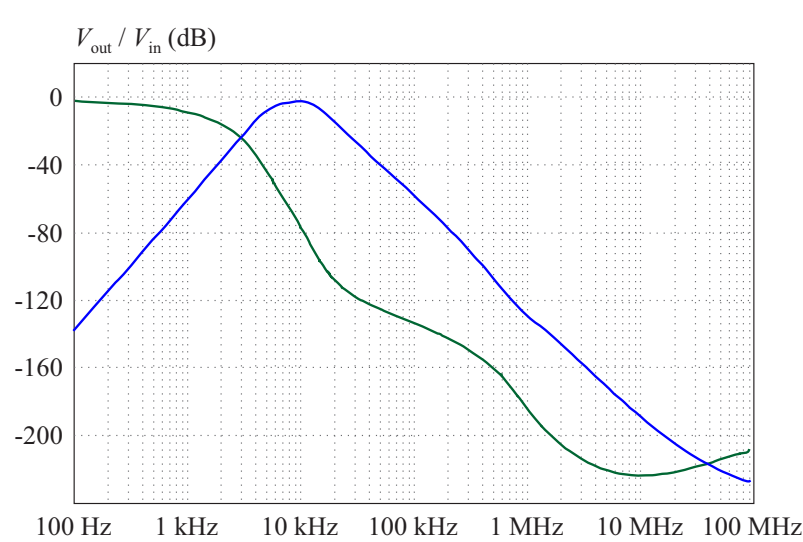

Fig. 7. Magnitude and phase characteristics of designed filter by SPICE program

Table 1. Butterworth coefficients

\begin{tabular}{lcc} 
Section & $F_{0}$ & $Q$ \\
\hline 1 & 1 & 0.541 \\
2 & 1 & 1.306 \\
\hline
\end{tabular}

Consider as equivalent LP-SK filter first, the theoretical transfer is $K 3=1+R_{\mathrm{A}} / R_{\mathrm{B}}=1.28$ where, $R_{\mathrm{A}}, R_{\mathrm{B}}$ are computed from Tab.1. For section No.4 transfer is $K_{4}=1+R_{10} / R_{11}=2.69$

- Capacitors and resistors of Huelsmann LP filter. Because in Huelsman LP-filter and in LP-SK filter the operation amplifier as voltage follower is not used, it is necessary to correct its attenuation. Therefor LP section amplification $K=K_{3} K_{4}=3.17$ and resistor $R_{8}$ in (SK-LP) must be substituted by the voltage divider $R_{81}, R_{82}$

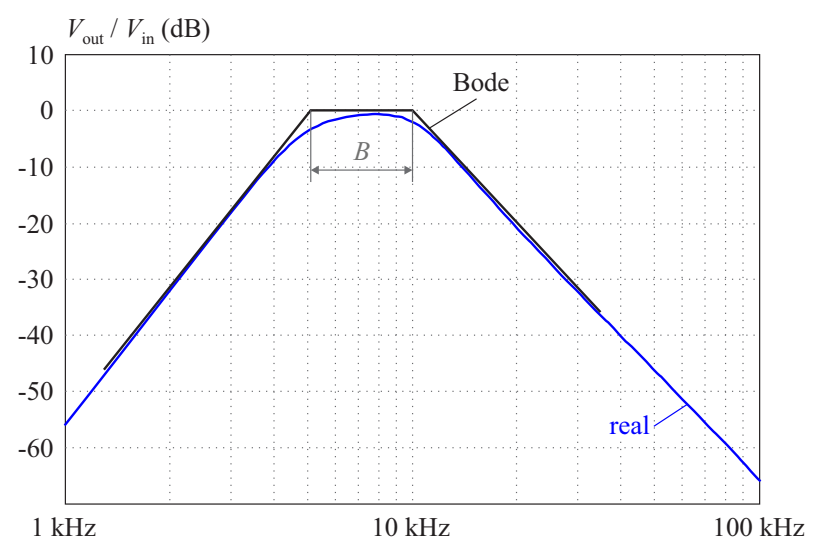

Fig. 8. Detail of magnitude characteristic by SPICE program and the 'hand-drawn' Bode plot $f_{\max }-f_{\min }=5 \mathrm{kHz}$

Final circuit diagram after all corrections with voltage divider is shown in Fig. 6, where are shown the values of all elements as well.

\section{Result of the simulation}

Simulation by SPICE verified this calculated solution. OpAmp LF155 ( GBP $=2.5 \mathrm{MHz})$ was used for these simulations. The decreasing in the attenuation at high frequencies did not occur for output corresponded to output node. The magnitude characteristic of designed filter is depicted in Fig. 7. As shown, in the frequency band from $100 \mathrm{~Hz}$ to $50 \mathrm{MHz}$, proposed solution is an ideal case of the frequency response of the band-pass filter.

Magnitude and phase characteristics of designed filter are depicted in Fig. 8. As can be seen, the phase characteristic has a course typical of the $\mathrm{BP} / \mathrm{SK}$ filter in the passband of frequencies. 
$L$

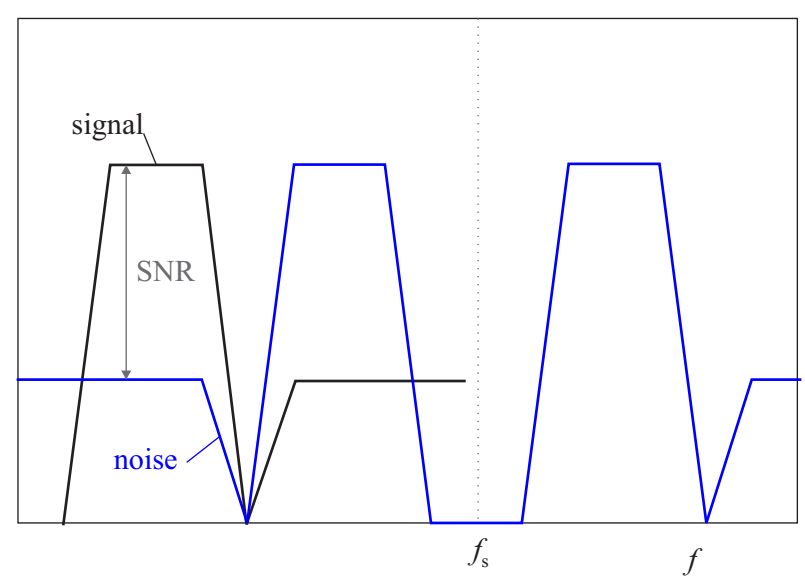

Fig. 9. The noise as product of the non ideal magnitude SK-HP and SK-LP antialiasing filter characteristics

Irregularity during the phase characteristic for frequencies above $10 \mathrm{MHz}$ is not significant, because the signal is attenuated by $200 \mathrm{~dB}$ ( $i e$ it is practically equal to zero). Detail of this magnitude characteristic of designed filter in narrow frequency band between $1 \mathrm{kHz}$ to $100 \mathrm{kHz}$, ie around the passband, is shown in Fig. 9. The Bode plot is shown as well.

\section{Discussion}

Finite output conductance in coincidence with a conductance of other circuit elements give to the frequencyindependent voltage divider (Fig. 4, 5) a non zero voltage at the output node for described biquads. Thus, if the output voltage is required equal to zero, then some of the internal voltage $\mathrm{V}$, see Fig. 5, must be equal to zero, too. Only, in this case, the filter stays in normal mode over a transient frequency of the used operational amplifier. In other cases, the decrease of the attenuation leads to an increase the noise interferring the useful signal, as shown in Fig. 10. Thus the signal-noise-to-ratio (SNR) is decreased as well. Note that in real conditions, the value of $-240 \mathrm{~dB}$ is unattainable, it is limited by the noise of real elements in the circuit.

\section{Conclusion}

The design of a band-pass filter is described, the proprieties of which, ie the course of the magnitude characteristic, are close to ideal. The filter consists of three SK biquads type and only one $\mathrm{H}$ type, so the advantageous properties of the SK type remain. This filter is suitable as an antialiasing for preprocessing of discrete-time frequency limited signals [9]. Calculated proposed result is verified by SPICE simulation.

\section{REFERENCES}

[1] R. Mancini, Op Amps For Everyone Design Reference, Texas Instruments, 2002.

[2] J. Punčocháŕ, "Low Pass Filters Sallen and Key With Real Operational Amplifiers", Elektrorevue 10, 2005, pp. 1-13.

[3] J. Bičak, M. Leipert, and M. Vlček, A Linear Circuits and Systems, CTU publisher, Prague, 2007.

[4] T. Dostál and K. Vrba, The Electric Filters, PC-DIR, Brno, 1997.

[5] J. Dostál, Operational Amplifiers, BEN publisher, Prague, 2006.

[6] D. Biolek, Solving Electronic Circuits, BEN publisher, Prague, 2004.

[7] W. Jung, Op Amp Applications Handbook, Elsevier, Oxford, UK, 2005, pp. 307-419.

[8] J. Čajka, and B. Kvasil J. Theory of Circuits, Linear and Linearized Circuits Praha/Bratislava (CS): SNTL/ALFA, 1979.

[9] S. Winder, Analog and Digital Filter Design, 2 nd ed, Woburn, USA, 2002, pp125-241.

Received 28 June 2021 